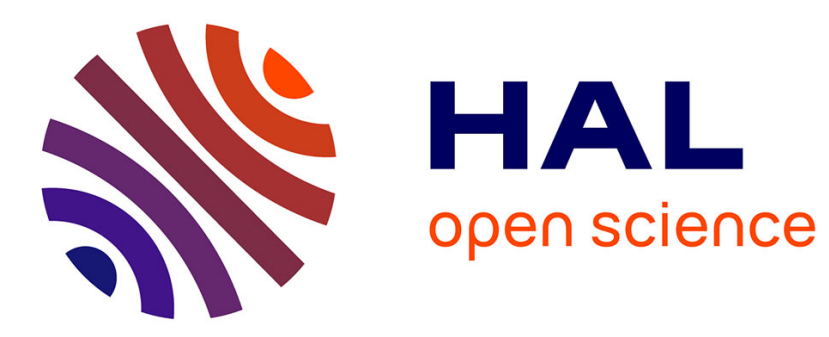

\title{
Weak localization of waves
}

E. Akkermans, R. Maynard

\section{- To cite this version:}

E. Akkermans, R. Maynard. Weak localization of waves. Journal de Physique Lettres, 1985, 46 (22), pp.1045-1053. 10.1051/jphyslet:0198500460220104500 . jpa-00232936

\section{HAL Id: jpa-00232936 https://hal.science/jpa-00232936}

Submitted on 1 Jan 1985

HAL is a multi-disciplinary open access archive for the deposit and dissemination of scientific research documents, whether they are published or not. The documents may come from teaching and research institutions in France or abroad, or from public or private research centers.
L'archive ouverte pluridisciplinaire HAL, est destinée au dépôt et à la diffusion de documents scientifiques de niveau recherche, publiés ou non, émanant des établissements d'enseignement et de recherche français ou étrangers, des laboratoires publics ou privés. 
Classification

Physics Abstracts

$42.20-63.50-71.55 \mathrm{~J}$

\title{
Weak localization of waves
}

\author{
E. Akkermans and R. Maynard (*) \\ Centre de Recherches sur les Très Basses Températures, \\ C.N.R.S., BP 166 X, 38042 Grenoble Cedex, France \\ $\left.{ }^{*}\right)$ and Université Scientifique et Médicale de Grenoble, France
}

(Reçu le 20 juin 1985, accepté le 24 septembre 1985)

\begin{abstract}
Résumé. - La localisation faible des ondes est obtenue à partir d'une théorie de diffusion multiple cohérente. Cela conduit à une augmentation de la section efficace différentielle dans la direction de rétrodiffusion. Celle-ci se manifeste de façon macroscopique par une valeur deux fois plus grande de l'intensité rétrodiffusée dans un cône très étroit autour de la direction d'incidence, d'ouverture angulaire $\phi_{\mathrm{c}}=\frac{\lambda}{l} \cdot \frac{\theta(\Omega)}{\tau}$, où $\theta(\Omega)$ est le temps de résidence. Les corrections aux coefficients de transport sont alors obtenues à deux et trois dimensions à partir de $\phi_{c}$. Un développement en $\varepsilon$ autour de la dimension critique inférieure $d_{\mathrm{c}}=2$ permet alors d'obtenir un seuil de localisation pour les ondes à $d>2$ et le comportement critique associé. On discute enfin de différentes situations expérimentales permettant d'observer à la fois le cône de rétrodiffusion et les exposants critiques.
\end{abstract}

\begin{abstract}
The weak localization of waves is formulated in terms of coherent multiple scattering theory. This leads, in the backscattering direction, to an enhancement of the differential cross-section. It manifests itself macroscopically by the doubling of the backscattering intensity in a narrow cone of width $\phi_{\mathrm{c}}=\frac{\lambda}{l} \cdot \frac{\theta(\Omega)}{\tau}$, where $\theta(\Omega)$ is the residence time around the incident direction. The corrections to transport coefficients are then derived both in two and three dimensions in terms of $\phi_{c}$. An $\varepsilon$-expansion around the lower critical dimension $d_{\mathrm{c}}=2$ is then performed and leads to a wave localization threshold for $d>2$ around which the critical behaviour is studied. Different kinds of experimental situations leading to the observation of this backscattering cone and critical exponents are then discussed.
\end{abstract}

\section{Introduction.}

The localization problem is now well-known to be an enormous and very dissymmetric hydra. While its electrical aspect, i.e. localization in electronic systems concerns a great number of solid state physicists, its non-electronic counterpart, for wave propagation in disordered media has been neglected until very recently. Most of theoretical predictions were limited to transport coefficients of metals as magnetoresistance of impure metals, temperature dependence of electrical conductivity, etc. The experimental confirmation of these predictions establishes the theory of weak localization on a strong basis. However, it is legitimate to ask if more direct observation of this general phenomenon might be envisaged in the field of wave scattering in random media. 
Recent progress in the interpretation of the weak localization phenomenon as a constructive interference effect shows that it only depends on the wave nature of the excitation and neither on quantum aspects nor on the nature of the statistics. To first order in $\lambda / l$, where $\lambda$ is the wave length and $l$ the elastic mean free path, this interference effect leads to a large anisotropy of the differential cross-section in the vicinity of the backscattering direction. We have found that inside a cone with an aperture of the order $\lambda / l$, the backscattered intensity is twice the value obtained within the standard incoherent multiple scattering approximation. Moreover, the specificity of this effect also appears in the power law time dependence of the backscattered echo from an incident pulse instead of exponential relaxation for the incoherent wave. A direct consequence of this constructive interference is the reduction of the radiation diffusion coefficient as in the case of electrons. This reduction can lead to a complete vanishing of the diffusion constant for $\lambda \lesssim l$. This conclusion is supported by a simple multiplicative renormalization group approach in $2+\varepsilon$ dimension which produces the critical exponents near the threshold. Finally, the sensitivity of the coherence effect to different dephasing processes is analysed, mainly those associated with confined geometry and moving impurities.

In this Letter, we attempt to present the weak localization phenomenon of waves in 2 and 3 dimensions in a somewhat transparent way. Some of the results established here are well-known : time-dependence of the response (echo) to an incident pulse [1], dephasing process due to impurity motion [2]. However some of our results are new :

i) Critical cone for coherent backscattering $\phi_{\mathrm{c}}=\frac{\lambda}{l} \cdot \frac{\theta(\Omega)}{\tau}$ (where $\Omega$ is the frequency bandwith of the backscattered wave and $\tau$ the elastic relaxation times).

ii) Preliminary results on the frequency threshold $\omega^{*}$ for localization of waves due to Rayleigh scattering.

iii) Critical behaviour of diffusion constant near $\omega^{*}$, and

iv) Dephasing effect associated with restricted geometry. We hope that this simple presentation will motivate new experiments in these fields.

\section{Incoherent and coherent multiple scattering : the factor 2 !}

We consider the problem of the propagation of an incident plane wave in an inhomogeneous medium with a density $\rho$ of fixed impurities described by a linear wave equation. We assume that these impurities act as pure elastic scatterers and that no absorption of the radiation results from this scattering. This problem can be defined in terms of several characteristic lengths. First, the wavelength $\lambda$ of the incident field, and second, the mean distance $\rho^{-1 / d}$ between impurities ( $d$ being the space dimensionality). In the limit $\lambda \ll \rho^{-1 / d}$, the transport of the incoherent field intensity through the medium can be described in terms of a Boltzmann-like equation leading to the elastic mean free path $l$. Now the two characteristic lengths of the inhomogeneous medium are $\lambda$ and $l$ for an incident wave which are functions of the frequency $\omega$.

Let us define the quantity $p\left(\hat{\mathbf{s}}, \hat{\mathbf{s}}^{\prime}\right)$ as the probability for the incoming field in direction $\hat{\mathbf{s}}$ to be scattered in direction $\hat{\mathbf{s}}^{\prime}$ ( $\hat{\mathbf{s}}$ and $\hat{\mathbf{s}}^{\prime}$ being unit vectors). Under stationarity conditions, $p\left(\hat{\mathbf{s}}, \hat{\mathbf{s}}^{\prime}\right)$ is given by

$$
p\left(\hat{\mathbf{s}}, \hat{\mathbf{s}}^{\prime}\right)=\frac{4 \pi}{\sigma_{\mathrm{t}}}\left|\phi\left(\hat{\mathbf{s}}, \hat{\mathbf{s}}^{\prime}\right)\right|^{2}
$$

where $\sigma_{\mathrm{t}}$ is the total scattering cross-section and $\phi\left(\hat{\mathbf{s}}, \hat{\mathbf{s}}^{\prime}\right)$ the scattering amplitude. The expression of $p\left(\hat{\mathbf{s}}, \hat{\mathbf{s}}^{\prime}\right)$ can be derived from the vertex function associated to the two propagator correlation function $C\left(\mathbf{r}_{1}, \mathbf{r}_{2} ; \mathbf{r}_{1}^{\prime}, \mathbf{r}_{2}^{\prime}\right)=\left\langle G^{\mathbf{R}}\left(\mathbf{r}_{1}, \mathbf{r}_{2}\right) G^{\mathbf{A}}\left(\mathbf{r}_{1}^{\prime}, \mathbf{r}_{2}^{\prime}\right)\right\rangle, G^{\mathbf{R}}$ and $G^{\mathbf{A}}$ being respectively retarded 
and advanced Green functions of the free propagating field, or from its Fourier transform $C\left(\mathbf{p}, \mathbf{p}^{\prime} ; \mathbf{q}, \mathbf{q}^{\prime}\right)$ by :

$$
p\left(\hat{\mathbf{s}}, \hat{\mathbf{s}}^{\prime}\right)=C\left(k \hat{\mathbf{s}}, k \hat{\mathbf{s}}^{\prime} ; k \hat{\mathbf{s}}, k \hat{\mathbf{s}}^{\prime}\right) .
$$

There are two classes of diagrams for multiple scattering :

- The ladder diagrams (Fig. 1a) which give the incoherent contribution $p_{\mathrm{L}}$ to the scattering probability.

- The crossed diagrams (Fig. 1b) describing the coherent contribution $p_{\mathrm{C}}$ to the probability. Then, we have :

$$
p\left(\hat{\mathbf{s}}, \hat{\mathbf{s}}^{\prime}\right)=p_{\mathbf{L}}\left(\hat{\mathbf{s}}, \hat{\mathbf{s}}^{\prime}\right)+p_{\mathbf{C}}\left(\hat{\mathbf{s}}, \hat{\mathbf{s}}^{\prime}\right)
$$

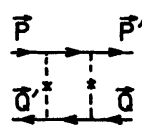

(a)

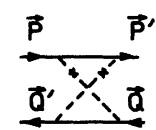

(b)

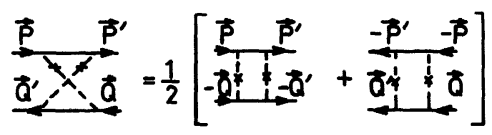

(c)

Fig. 1. - (a) Ladder diagram for incoherent multiple scattering; (b) crossed diagram for coherent multiple scattering and (c) identity relation for crossed diagrams in terms of ladder diagrams.

By using the time reversal symmetry of the scattering potential of the impurities, the crossed diagrams can be expressed at each order of the multiple scattering expansion by the equality of figure 1c. More precisely,

$$
p_{\mathrm{C}}\left(\hat{\mathbf{s}}, \hat{\mathbf{s}}^{\prime}\right)=\frac{1}{2}\left[C\left(k \hat{\mathbf{s}}, k \hat{\mathbf{s}}^{\prime} ;-k \hat{\mathbf{s}}^{\prime},-k \hat{\mathbf{s}}\right)+C\left(-k \hat{\mathbf{s}}^{\prime},-k \hat{\mathbf{s}} ; k \hat{\mathbf{s}}, k \hat{\mathbf{s}}^{\prime}\right)\right]
$$

Note that for the special situation of backscattering, $\hat{\mathbf{s}}^{\prime}=-\hat{\mathbf{s}}$

and

$$
p_{\mathrm{C}}(\hat{\mathbf{s}},-\hat{\mathbf{s}})=C(k \hat{\mathbf{s}}, k \hat{\mathbf{s}} ;+k \hat{\mathbf{s}}, k \hat{\mathbf{s}})=p_{\mathrm{L}}(\hat{\mathbf{s}},-\hat{\mathbf{s}})
$$

$$
p(\hat{\mathbf{s}},-\hat{\mathbf{s}})=2 p_{\mathbf{L}}(\hat{\mathbf{s}},-\hat{\mathbf{s}}) .
$$

Therefore, the total probability of backscattering $p(\hat{\mathbf{s}},-\hat{\mathbf{s}})$ including coherent and incoherent scattering is exactly twice the incoherent multiple scattering probability. This result is well known $[1,3]$ and our proof shows its full generality.

\section{Cone of backscattering and coherence bandwith.}

We have calculated the coherent backscattered intensity by the Bethe-Salpeter equation for the maximally crossed diagrams and the detail of this calculation will be reported elsewhere [4]. Here, we want to establish the main results of the diagrammatic expansion in a very simple and physical way following the presentation of G. Bergmann [1] for the weak localization : « time of flight experiment with conduction electrons ». Let us assume first that the propagation in this 
very inhomogeneous medium is such that the waves become depolarized rapidly over distances of the order of the mean free path $l$. The radiation fields will therefore be considered as scalar waves without polarization.

Assume that, at $t=0$, a pulse enters the medium with wave vector $k=2 \pi / \lambda$. The available final states for the elastic incoherent multiple scattering are located in the spherical shell of radius $k$ and thickness $1 / l$. The number of final states is given for isotropic scattering described by $l(d=2$ or 3) by

$$
2^{d-1} \pi k^{d-1} \cdot \frac{1}{l}
$$

At time $t$ later, the wave field explores a volume of linear size $\left(D_{0} t\right)^{1 / 2}$ where $D_{0}$ is the unperturbed diffusion coefficient. The smearing of the wave vector $\Delta k$ due to this confinement is given by $\left(D_{0} t\right)^{-1 / 2}$. The available states for the coherent backscattering is therefore given by $(\Delta k)^{d}$ from which we obtain the ratio of coherent backscattering intensity over the total number of scattered states surviving up to time $t$ :

$$
\frac{(\Delta k)^{d}}{2^{d-1} \pi k^{d-1} l^{-1}}=\frac{1}{(k l)^{d-1}} \cdot\left(\frac{\tau}{t}\right)^{d / 2} \quad(t \gg \tau) .
$$

This result has been already established [1] for $d=2$ : the long time tail of this echo originates in the multiple scattering processes of the incoming wave field of the pulse for a time $t$. All possible paths from $+\mathbf{k}$ to $-\mathbf{k}$ are taken into account in the infinite medium (for a restricted geometry such as a slab, a typical cut-off in time would describe the limitation in the number of multiple scattering events (see Sect. 5)). The classical analog of this result is the probability of return to the origin at time $t$ which involves all possible loops of a free random walk.

From scattering wave theory we know that the real part of the Fourier transform of time development of the echo is related to the two-frequency correlation function or equivalently to the coherence bandwith [5] (broadening of a pulse due to the random medium). Let us call $\theta(\Omega)$ this Fourier transform where $\Omega$ measures the frequency shift of the backscattered radiation. For vanishing $\Omega$, i.e. for $\Omega \tau \ll 1$, we obtain from equation (8) :

$$
\frac{\theta_{d}(\Omega)}{\tau} \approx\left\{\begin{array}{ll}
\text { cte } & d=3 \\
\ln \frac{1}{\Omega \tau} & d=2
\end{array} .\right.
$$

This result has a classical analog in the theory of the random motion of a particle. $\theta_{d}(\Omega)$ is the residence time of the particle at the origin during a time interval of order $1 / \Omega$. In 3 dimensions, the residence time of the particle at the origin is finite and of order $\tau$ while, due to the recurrent nature of the random walk in $d=2$, it diverges although it explores all the space. Then, the logarithmic divergence of $\theta(\tau)$, which has been settled by various authors [6], is a signature of the two-dimensional localization of waves. From equations (8) and (9) we have access to the spectral relative intensity of coherent backscattering which is proportional to

$$
\left(\frac{\lambda}{l}\right)^{d-1} \frac{\theta_{d}(\Omega)}{\tau} .
$$

This result has a direct implication for the differential cross-section. Let us call $\sigma_{\mathrm{i}}$ the incoherent contribution to the differential cross-section in the direction of backscattering. We have previously established that the intensity of coherent backscattering is twice the intensity of incoherent backscattering. Then for the backscattering direction, the coherent differential cross-section $\sigma_{c}$ is just twice $\sigma_{\mathrm{i}}$ at each frequency. Expression (10) allows us to go beyond this property, since 
it gives the value of the total cross-section for backscattering. It defines a cone in such a way that :

$$
\text { solid angle } \propto \phi^{d-1} \approx\left(\frac{\lambda}{l}\right)^{d-1} \cdot \frac{\theta_{d}(\Omega)}{\tau}
$$

where $\phi^{d-1}$ is the small angular aperture of the cone in $d$ dimensions with $\phi$ the angle of the cone in the direction of the backscattering defined in figure 2. From equation (11) one obtains the characteristic angular aperture $\phi_{c}$ of the cone for the coherent backscattered intensity :

$$
\phi_{\mathrm{c}}= \begin{cases}a \frac{\lambda}{l} & d=3 \\ b \frac{\lambda}{l} \ln \frac{1}{\Omega \tau} & d=2\end{cases}
$$

where $a$ and $b$ are numerical constants we identify below.

The main physical result is contained in the dimensionless ratio $\lambda / l$ of the wavelength over the elastic mean free path of the radiation. This result of a perturbative nature is valid not only for $\lambda / l \ll 1$ but also, in 2 dimensions for $\lambda / l \cdot \ln 1 / \Omega \tau \ll 1$. This condition limits the validity of this formula to the low frequency range : it is precisely the range of validity of the perturbation theory for the weak localization in 2 dimensions. We believe that the singularity at $\Omega=0$ is non-physical and needs a scaling treatment as developed in section 5 where, in the singular limit $\Omega \rightarrow 0$, the dimensionless ratio $\lambda / l$ is renormalized and provides a term which suppresses the logarithmic divergence.

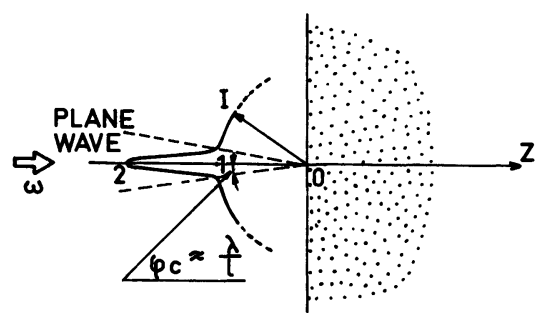

Fig. 2. - Profile intensity in the backscattering direction. $\phi_{c} \cong \lambda / l$ is the characteristic angle of the cone inside of which coherent intensity is backscattered.

\section{Correction to the diffusion coefficient.}

It is well known in transport theory [7] that the mean free path can be directly deduced from the differential cross-section $\sigma(\phi)$ by the following formula :

$$
\begin{cases}\frac{1}{l}=\rho \int_{0}^{\pi}\left(\sigma_{\mathrm{i}}+\sigma_{\mathrm{c}}\right)(1+\cos \phi) 2 \pi \sin \phi \mathrm{d} \phi & d=3 \\ \frac{1}{l}=2 \rho \int_{0}^{\pi}\left(\sigma_{\mathrm{i}}+\sigma_{\mathrm{c}}\right)(1+\cos \phi) \mathrm{d} \phi & d=2\end{cases}
$$


where $1+\cos \phi$ is the geometrical term entering the transport theory due to the anisotropy of the relaxation process. Actually it weighs on the backscattering by an extra factor $1+\cos \phi \cong 2$. The additional contribution of the coherent backscattering will shorten the mean free path as compared to the incoherent contribution. For an isotropic scattering where $\sigma_{\mathrm{i}}$ is independent of $\phi$, this shortening of $l$ is better described by the reduction of the diffusion coefficient from the incoherent value $D_{0}$

$$
\begin{cases}D \cong D_{0}\left(1-\phi_{\mathrm{c}}^{2}\right) & d=3 \\ D \cong D_{0}\left(1-\frac{4}{\pi} \phi_{\mathrm{c}}\right) & d=2\end{cases}
$$

By comparing these expressions with exact results obtained by summing the infinite series of maximally crossed diagrams [4] we identify the numerical constants $a=3 / 4 \pi$ and $b=1 / 4 \pi$.

First it must be noticed that this diffusion coefficient is parametrized by the energy $\omega$ through $D_{0}$ and the ratio $\lambda / l$. As expected the contribution of the coherent backscattering reduces the diffusion coefficient and consequently all the transport coefficients by a term proportional to the characteristic angle $\phi_{\mathrm{c}}$. Here again, the validity of the present approximation of weak localization limits the correction to small value of $\phi_{\mathrm{c}}$. But nevertheless the question of the vanishing diffusion coefficient or equivalently the existence of an edge of localization for waves emerges from this context. Additional assumptions are then necessary to establish such a localization threshold. These are developed in the next section.

\section{Renormalization-group treatment of the perturbation expansion-scaling.}

The previous perturbation expansion of $D$ shows how disorder modifies the diffusion coefficient $D_{0}$. The form of the correction to $D$ for $d=2$ given by equations (12) and (14) can be rewritten as :

$$
D=D_{0}\left[1+\frac{\lambda}{l} \ln \left(\frac{\Omega}{\Lambda^{2}}\right)\right] .
$$

It strongly suggests the possibility of a scaling theory based on one parameter only $t_{0}=\lambda / l$ and with a characteristic frequency cut-off $\Lambda^{2}=1 / \tau$. This scaling behaviour can be obtained within the usual one-parameter renormalization group treatment [8].

The divergence in $d=2$ of the corrections to $D$ at each order of perturbation [6] shows that $d=2$ is the lower critical dimension for the problem of localization. It allows us to perform an $\varepsilon$ expansion in $d=2+\varepsilon$ dimension. Consider the renormalization of the vertex $\Gamma_{0}(t, \Omega)$ defined by $D=D_{0} \Gamma_{0}(t, \Omega)$, according to

$$
\Gamma(t, \Omega)=Z(\Lambda) \Gamma_{0}(t, \Omega) .
$$

The correction to $D$ to first order in $t_{0}$ is proportional to $t_{0}(\Omega \tau)^{\varepsilon / 2}$. Then, if we rescale the frequency $\Omega$ according to $\Omega / \Lambda^{2}$, the coupling constant scales as $\Lambda^{\varepsilon} t(\Lambda)$. This leads to the following relation for the scaling variables :

$$
\left\{\begin{array}{l}
t_{0}=\Lambda^{\varepsilon} Z^{-1}(\Lambda) t(\Lambda) \\
\Omega=\Lambda^{2} Z(\Lambda) \Omega(\Lambda)
\end{array} .\right.
$$

The identification of both the standard $1 / \varepsilon$ expansion of $Z$ and $\Gamma_{0}$ to first order in $1 / \varepsilon$ gives :

$$
Z(t)=1+2 \pi \frac{t}{\varepsilon}
$$


Then the low frequency behaviour of $D(\Omega)$ is obtained by taking the limit $\Lambda \rightarrow \infty$, where the scale invariance of the renormalized vertex $\Gamma$ leads to the following renormalization group equation for $\Gamma_{0}$ :

with

$$
\left[A(\Omega) \frac{\partial}{\partial \Omega}+\beta(t) \frac{\partial}{\partial t}-\eta(t)\right] \Gamma_{0}=0
$$

$$
A(\Omega)=\frac{\partial \Omega}{\partial \ln \Lambda^{\prime}}, \quad \beta(t)=\frac{\partial t}{\partial \ln \Lambda} \quad \text { and } \quad \eta(t)=\frac{\mathrm{d} \ln Z^{-1}}{\mathrm{~d} \ln \Lambda} .
$$

Studying the critical behaviour of $D$ when $\Omega \rightarrow 0$ is equivalent to study that of $t(\Lambda)$ in the same limit $\Lambda \rightarrow \infty$. Now, we have :

$$
\beta(t)=\varepsilon t\left(1+\frac{\partial \ln Z}{\partial \ln t}\right)^{-1}
$$

and with the aid of equation $(18): \beta(t)=t(\varepsilon-2 \pi t)$.

Then, for $d>2$, the trivial fixed point $t=0$ exchanges its stability with the non-trivial solution $t^{*}=\varepsilon / 2 \pi$ which is stable for $\Lambda \rightarrow \infty$ as shown in figure 3 . This fixed point corresponds to a mobility edge for $d>2$ in the spectrum parametrized by $\omega$ between delocalized modes at low energies and localized modes for $\omega>\omega^{*}$ where $t\left(\omega^{*}\right)=t^{*}$. An equivalent result has been obtained [9] by means of a mapping of the localization problem to a non-linear $\sigma$-model.

The renormalization group equation (19) allows us to obtain the frequency dependence of $D$ at the critical point $\omega^{*}$. We then have

$$
A\left(\Omega, t^{*}\right)=\Omega(2+\varepsilon) \text { and } \eta\left(t^{*}\right)=-\varepsilon
$$

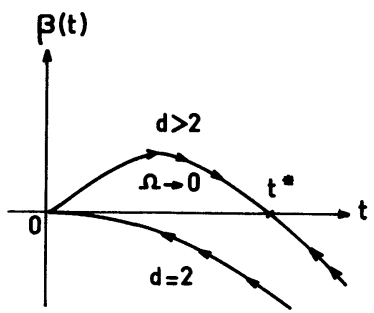

Fig. 3. - Plot of $\beta(t) v s$. $t$ for $d>2$ and $d=2 . \beta(t)$ represents the variation of the coupling constant $t=\lambda / l$ with the frequency scale and $t^{*}$ the fixed point corresponding to the localization transition.

and the resolution of equation (19) gives :

$$
D\left(\Omega, \omega^{*}\right) \underset{\Omega \rightarrow 0}{\sim} D_{0} \Omega^{d-2 / d}
$$

and

$$
D(0, \omega) \underset{\omega \rightarrow \omega^{*}}{\sim}\left|\omega^{*}-\omega\right|^{1 / d}
$$

which confirms the vanishing of $D$ at $\omega^{*}$ for $\Omega \rightarrow 0$ and gives the behaviour of $D$ at zero frequency near the localization threshold. It is then possible to derive a complete scaling theory for the Anderson transition [10] for wave propagation in a random medium, without knowing the underlying field theory. 


\section{Discussion and conclusion.}

There are two main results in this study. First, the characteristic angles $\phi_{c}$ in equation (12) of the spectral intensity cone of backscattering are obtained in 2 and 3 dimensions, in terms of the ratios $\lambda / l$ and $\theta(\Omega) / \tau$. Second the appearance of a threshold of localization as well as the critical behaviour of diffusion coefficient around the critical point are discussed by a direct scaling argument.

The possible existence of this cone would be a direct confirmation of the concepts of weak localization and calls for new experiments. In view of measurements we must discuss various phase breaking processes for coherent backscattered radiation. To any kind of dephasing process we can associate a characteristic cut-off time $\tau_{c}$ beyond which the contribution of the multiple scattering path to the coherent backscattering vanishes. This is well known for electrons where the inelastic scattering time introduces a natural cut-off to the correction of electrical conductivity. The general method for handling $\tau_{\mathrm{c}}$ in the theory is to cut-off time development of the echo above $\tau_{\mathrm{c}}$. In the limit of very low frequency $\Omega \tau_{\mathrm{c}} \ll 1$, the dephasing correction is simply evaluated for $\tau_{\mathrm{c}} \gg \tau$ :

$$
\left\{\begin{array}{ll}
\phi_{\mathrm{c}}=a \frac{\lambda}{l}\left(1-\sqrt{\frac{\tau}{\tau_{\mathrm{c}}}}\right) & d=3 \\
\phi_{\mathrm{c}}=b \ln \frac{\tau_{\mathrm{c}}}{\tau} & d=2
\end{array} .\right.
$$

In 2 dimensions the divergence for $\Omega \rightarrow 0$ is indeed suppressed : $\tau_{\mathrm{c}}^{-1}$ is the lower cut-off frequency for scaling invariance. In 3 dimensions three types of dephasing processes could be encountered for weak localization of waves : absorption with a characteristic time $\tau_{\mathbf{a}}$, impurity motion for which $\tau_{\mathrm{c}}=\tau_{\lambda}$ is the time of motion of the impurity over a distance $\lambda$, and finally finite geometry effect. The correction $\tau_{\lambda}$ has already [2] been discussed : it is weak since it involves the ratio of the impurity average velocity over the velocity of light or sound (for ultrasound this correction could be sizeable). More precisely, we find

$$
\frac{\tau}{\tau_{\lambda}}=\frac{v_{\mathrm{i}}}{c} \cdot \frac{l}{\lambda}
$$

for a dilute gas of impurities with velocity $v_{\mathbf{i}}$, while for Brownian motion (diffusion coefficient $D_{\mathbf{i}}$ ) the correction is still weaker : $\tau_{\lambda}=\lambda^{2} / D_{\mathrm{i}}$. The case of impurities imbedded in a slab of thickness $\mu$ can be simply treated if the incoming wave from the left is totally transmitted outside on the right. In this case the cut-off time $\tau_{c}$ is just the time for the radiation to diffuse up to a length scale of order $\mu$ :

$$
\tau_{\mathrm{c}} \simeq \tau_{\mu} \simeq \frac{\mu^{2}}{D}
$$

The finite geometry correction at $d=3$ is therefore $\left(\tau / \tau_{\mu}\right)^{1 / 2}=l / \mu$, i.e. the ratio of $l$ over $\mu$.

The problem of localization threshold deserves to be discussed in the context of the Rayleigh scattering [5] of electromagnetic waves. From a standard formula for the Rayleigh mean free path $l(\omega) \approx \omega^{-4}$ we define in $d=3$ :

$$
\frac{\lambda}{l}=\left(\frac{\omega}{\omega_{\mathrm{R}}}\right)^{3}
$$

with

$$
\omega_{\mathrm{R}}=\left(\frac{16}{3}\right)^{1 / 3} n_{\mathrm{i}}^{-1 / 3} \frac{c}{a^{2}}\left|\frac{\varepsilon_{\mathrm{r}}-1}{\varepsilon_{\mathrm{r}}+2}\right|^{2 / 3}
$$


where $a$ is the radius of spherical particles of relative dielectric constant $\varepsilon_{\mathrm{r}}$ (involving the condition of validity : $\lambda \gg a$ ). The frequency threshold $\omega^{*}$ is given at $d=3$ by the extrapolated expression (21) for $2+\varepsilon=3$. From this relation we obtain a threshold $\omega^{*}=0.54 \omega_{\mathrm{R}}$. A very similar result [4] can be obtained for sound waves with Klemens scattering on mass impurities : $\omega^{*}=0.54 \omega_{\mathrm{K}}$ where $\omega_{\mathrm{K}}=0.14 \sigma_{\mathrm{M}}^{-2 / 3} \omega_{\mathrm{D}}$.

The approach of the threshold should correspond in both cases to situation of very dense concentration of defects : $n_{\mathrm{i}} a^{3} \rightarrow 1$ for light and each atom considered as mass defect for phonons. The realization or observation of such situations would produce blackhole for high frequency waves since no intensity could emerge from such dirty medium despite the fact that no absorption occurs in the scattering on impurities.

\section{Acknowledgments.}

It is a pleasure to thank Prof. A. Lagendijk and Dr. P. E. Wolf for valuable discussions, Dr. R. Rammal for a critical reading of the manuscript.

\section{References}

[1] Bergmann, G., Phys. Rep. 107 (1984) 1.

[2] Golubentsev, A. A., Sov. Phys. JETP 59 (1984) 26.

[3] See Khmelnitskit, D. E., Physica B + C 126 (1984) 235.

[4] Akmermans, E. and Maynard, R., to be published.

[5] Ishimaru, A., Wave Propagation and Scattering in Random Media (Academic Press, New York) 1978.

[6] Gorkov, L. P., LARKIN, A. I. and KhMelNitSKII, D. E., JETP Lett. 30 (1979) 228.

[7] Landau, L. D. and Lifshitz, E. M., Course of Theoretical Physics, Physical Kinetics (Pergamon Press) 1981.

[8] Wilson, K. G. and Kogut, J. B., Phys. Rep. 12 (1974) 75.

Brezin, E., Le Guillou, J. C. and Zinn-Justin, J., Phase Transitions and Critical Phenomena, Vol. 6, ed. C. Domb and M. S. Green (London : Academic Press) 1976.

[9] John, S., Sompolinski, H. and StePhen, M. J., Phys. Rev. B 27 (1983) 5592.

[10] Abrahams, E., Anderson, P. W., Licciardello, D. C. and Ramakrishnan, T. V., Phys. Rev. Lett. 42 (1979) 673. 